
in the spotight

$\mathrm{P}$ rovision of structured weight-loss programs to overweight or obese individuals can lead to appreciable and sustained reductions in body weight, as well as conferring tangible benefits on cardiovascular risk factors, report researchers in the Journal of the American Medical Association.

Obesity continues to present a major public health issue but opinion is divided on how best to tackle the problem. Although commercial weightloss programs are extremely popular, very little scientifically rigorous data is available on their efficacy and safety. Cheryl Rock and colleagues, therefore, conducted a proof-of-principle study to determine whether or not one particular commercial program (Jenny Craig, Carlsbad, CA) could safely promote weight loss when followed as advised by the manufacturer.

Rock et al. recruited 442 women with overweight or obesity (BMI $25-40 \mathrm{~kg} / \mathrm{m}^{2}$ )

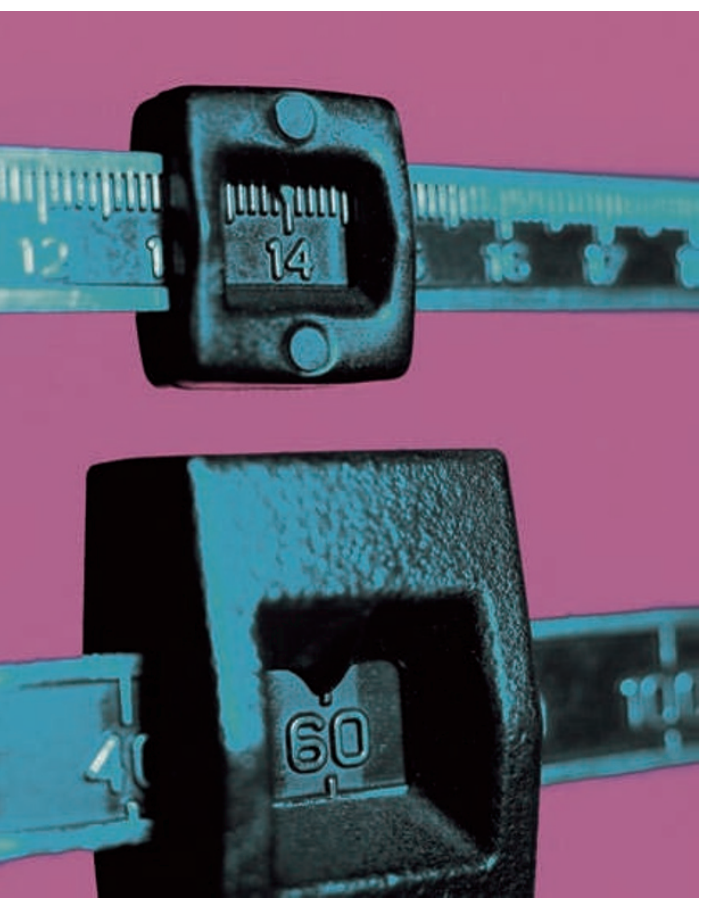

to participate in their randomized, controlled trial of the Jenny Craig program versus usual care at four sites in the USA. The study was limited to women, as men generally comprise the minority of individuals who enroll in commercial weight-loss programs outside the setting of a clinical trial.

Women assigned to the Jenny Craig program received all program materials, including prepackaged, prepared meals, free of charge. The program comprised one-to-one weekly counseling (at a Jenny Craig center or by telephone); dietary advice and meal plans; and a regular exercise regimen. Usual care comprised two in-depth, in-person counseling sessions, plus monthly contacts with a dietetics professional.

Mean weight loss from baseline at 12 months was $10.1 \mathrm{~kg}$ (center-based group), $8.5 \mathrm{~kg}$ (telephone-based group) and $2.4 \mathrm{~kg}$ (usual care). After 24 months, $62 \%$ of center-based participants and $56 \%$ of telephone-based participants had maintained a weight loss of $\geq 5 \%$. Levels of C-reactive protein (a surrogate marker of metabolic disease) were also reduced in the two Jenny Craig groups. These results raise the possibility that the cost of providing free access to commercial weight-loss programs might be a small price to pay for increased retention and improved outcomes, particularly when compared with other (more expensive) options for treating obesity, such as bariatric surgery.

In a second study, researchers evaluated the effect of providing a lifestyle modification program to a specific subgroup of individuals with obesity. "We decided to focus this project on severe obesity as we felt that this was an underinvestigated area," explains first author Bret Goodpaster, an Associate Professor of Medicine at the University of Pittsburgh, PA. Lifestyle modification is conventionally thought to be an ineffective treatment option for the severely obese; however, the investigators' rationale was that even modest weight loss (5-10\% of initial body weight) might still be associated with clinically relevant health benefits in this group.

Goodpaster et al. enrolled 130 adults with either class II (BMI 35.0-39.9 kg/m²) or class III (BMI $\geq 40.0 \mathrm{~kg} / \mathrm{m}^{2}$ ) obesity. The participants underwent an intensive, 1-year lifestyle counseling program that focused on diet and physical activity. One group received both the dietary and exercise components of the intervention for the entire study period, whereas, in a second group, initiation of the exercise component was delayed for 6 months.

Statistically significant weight loss was observed in both groups at 6 and 12 months, particularly among individuals with class III obesity. Of note, almost $30 \%$ of the participants had achieved a weight loss of $>10 \%$ by study end. Cardiometabolic risk factors-such as visceral adiposity, insulin resistance, hepatic steatosis and blood pressure-were also improved in both groups.

"The key message is that severely obese adults can respond favorably to behaviorbased diet and exercise programs," Goodpaster comments. "So, although bariatric surgery will continue to play an important part in combating the obesity epidemic, lifestyle programs, if effectively implemented, can and should be considered."

\section{Vicky Heath}

Original articles Rock, C. L. et al. Effect of a free prepared meal and incentivized weight loss program on weight loss and weight loss maintenance in obese and overweight women: a randomized controlled trial. JAMA 304, 1803-1810 (2010) | Goodpaster, B. H. et al. Effects of diet and physical activity interventions on weight loss and cardiometabolic risk factors in severely obese adults: a randomized trial. JAMA 304, 1795-1802 (2010) 